\title{
A high carbohydrate diet does not induce hyperglycaemia in a mitochondrial glycerol-3-phosphate dehydrogenase-deficient mouse
}

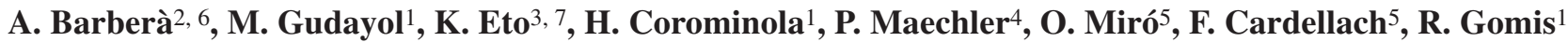 \\ ${ }^{1}$ Endocrinology and Nutrition Unit, Department of Medicine, Hospital Clinic/ \\ Institut d'Investigacions Biomèdiques August Pi i Sunyer (IDIBAPS), University of Barcelona, Barcelona, Spain \\ ${ }^{2}$ Laboratory of Metabolic Diseases, Rockefeller University, New York \\ ${ }^{3}$ Department of Metabolic Diseases, Graduate School of Medicine, University of Tokyo, Japan \\ ${ }^{7}$ CREST of Japan Science and Technology Corporation, Japan \\ ${ }^{4}$ Division of Clinical Biochemistry, University Medical Center, University of Geneva, Switzerland \\ ${ }^{5}$ Laboratory of Mitochondrial Research, Department of Internal Medicine, Hospital Clinic/ \\ Institut d'Investigacions Biomèdiques August Pi i Sunyer (IDIBAPS), University of Barcelona, Spain \\ ${ }^{6}$ Experimental Diabetes Laboratory, Hospital Clinic/Institut d'Investigacions Biomèdiques August Pi i Sunyer (IDIBAPS), \\ University of Barcelona, Spain
}

\begin{abstract}
Aims/hypothesis. The electrons of the glycolysis-derived reduced form of NADH are transferred to mitochondria through the NADH shuttle system. There are two NADH shuttles: the glycerol phosphate and malate-aspartate shuttle. Mice with a targeted disruption of mitochondrial glycerol-3-phosphate dehydrogenase, a rate-limiting enzyme of the glycerol phosphate shuttle, are not diabetic and have normal islet glucoseinduced secretion. In this study, we analyzed if environmental factors, such as a high carbohydrate diet could contribute to the development of Type 2 diabetes mellitus in mice with a specific defective genetic background.

Methods. The mice were fed with a high carbohydrate diet for 1 and 6 months, and several biochemical parameters were analysed. The mitochondrial respiratory activity was assayed by polarography; and the islet
\end{abstract}

function was studied by islet perifusion and pancreas perfusion.

Results. The high carbohydrate diet induced hyperglycaemia, hyperinsulinaemia, and islet hyperplasia in the wild-type and heterozygote mice. Activity of the respiratory chain complex I also increased in these mice. In contrast, these effects were not observed in the null mice fed with the diet; in addition, these null mice had an increased insulin sensitivity compared to wild-type mice.

Conclusion/interpretation. The phenotype of the mice with an impairment of NADH shuttles does not worsen when fed a high carbohydrate diet; moreover, the diet does not compromise islet function. [Diabetologia (2003) 46:1394-1401]

Keywords High carbohydrate diet, mitochondrial metabolism, mGPDH, beta cell, glycerol phosphate shuttle, insulin secretion.
Published online: 13 September 2003

(C) Springer-Verlag 2003

Corresponding author: R. Gomis MD PhD, Endocrinology and Nutrition Unit, Department of Medicine, Hospital Clinic/Institut d'Investigacions Biomèdiques August Pi i Sunyer (IDIBAPS), University of Barcelona, Villarroel 170, 08036 Barcelona, Spain E-mail: gomis@medicina.ub.es

Abbreviations: mGPDH, mitochondrial glycerol-3-phosphate dehydrogenase.

A. Barberà and M. Gudayol have contributed equally to this paper
In the beta cell, mitochondria exert a pivotal role in the regulation of glucose-induced insulin secretion, and mitochondrial activation is required for normal signal transduction [1]. There are at least two NADH shuttles: the glycerol-phosphate shuttle [2] and the malate-aspartate shuttle [3]. Both are essential contributors to redox traffic between the cytosolic and mitochondrial compartments. The glycerol-phosphate shuttle is thought to be a direct link in the metabolic pathway between glycolysis and mitochondrial oxidation, and has been reported to be most active in tissues 
that rapidly oxidize glucose, such as testis and pancreatic beta cells [4].

Mitochondrial glycerol-3-phosphate dehydrogenase (mGPDH) is a rate-limiting enzyme for the glycerol phosphate shuttle [5]. Activity of this enzyme has been reported to be low in a variety of animal models of diabetes such as neo-natal streptozotocin [6,7], Goto-Kakizaki [8, 9] and zucker diabetic fatty rats [10]. Moreover, similar results have been obtained from human islets from Type 2 diabetic patients [11]. Therefore, several authors have suggested that mGPDH could play an important role in the pathogenesis of Type 2 diabetes [11, 12, 13]. To determine the role of the glycerol-3-phosphate shuttle in glucose-induced insulin secretion, mice with a targeted disruption of the $m G P D H$ gene were generated $[14,15]$. $m G P D H^{--}$knockout mice are not diabetic, only when the activities of both shuttles are halted by the use of aminooxyacetate, to block the malate-aspartate shuttle, are insulin secretion, glucose metabolism, and calcium oscillations severely impaired $[16,17]$.

Type 2 diabetes is a multifunctional disease, in which both genetic and environmental factors are involved $[18,19]$. There are reports of previously nondiabetic knockout mice becoming diabetic when challenged with an environmental factor such as diet [20, 21]. Using $m G P D H$ knockout mice, we studied the effects of a high carbohydrate diet; analyzing whether a combination of an environmental factor such as a high carbohydrate diet and a genetic defect could contribute to the development of Type 2 diabetes.

\section{Material and methods}

Mice. The $m G P D H^{-1-}$ C57BL/6J mice were a gift from Dr. K. Eto from the Department of Metabolic Diseases, University of Tokyo (Tokyo, Japan). Principles of laboratory animal care were followed (EU and local government guidelines) and protocols were approved by the Animal Research Committee of the University of Barcelona. The mice were maintained at a constant temperature of $22^{\circ} \mathrm{C}$ with a fixed artificial light cycle (12-h light/dark cycle). Mice were given either a normal diet (Panlab A04 with 59\% carbohydrates, 17\% protein, and 3\% fat; and $317 \mathrm{Kcal} / 100 \mathrm{~g}$ ) or a high carbohydrate diet (ICN High Carbohydrate Diet with $70 \%$ carbohydrates, $19.5 \%$ protein, and $3 \%$ fat; and $397 \mathrm{Kcal} / 100 \mathrm{~g}$ ). At 2 months of age male mice were fed for either 1 or 6 months, with free access to both food and fluid. During the experiment food and fluid intake, body-weight gain, and glycaemia were monitored. At the end of the experiment, the mice were killed and plasma insulin, cholesterol, triglyceride and NEFA values were measured. Plasma triglycerides and cholesterol were measured spectrophotometrically by standard techniques (Bayer Diagnostics, Germany). Free fatty acids were measured by enzymatic colorimetric assay (Roche Diagnostics, Switzerland).

Assessment of glucose and insulin tolerance test. The intraperitoneal glucose tolerance test was carried out in male mice fasted for $16 \mathrm{~h}$, and glucose (150 mg/kg body weight) was administered intraperitoneally after anaesthesia with sodium pento- barbital $(60 \mathrm{mg} / \mathrm{kg})$. Blood was sampled from the tail vein before, and at 15, 30, 60, 120 and 180 min after glucose administration. Blood glucose concentrations were measured using a Glucometer Elite (Química Farmaceutica Bayer, Barcelona, Spain). Insulin plasma was also measured by ELISA method (Mercodia, Uppsala, Sweden). In the insulin tolerance test, human insulin $(0.75 \mathrm{U} / \mathrm{kg})$ was injected intraperitoneally in anaesthetized adult mice after fasting for $16 \mathrm{~h}$. Blood samples were taken from the tail vein.

Pancreatic islet isolation and insulin secretion. Islets from $m G P D H^{-/}, m_{G P D H}^{-/+}$and $m G P D H^{+/+}$were isolated by a modification of the method described previously [22]. In brief, $2 \mathrm{ml}$ of Hanks' solution containing collagenase (Roche Diagnostics, Switzerland) was injected into the duct; the swollen pancreas was removed and incubated at $37^{\circ} \mathrm{C}$ for $6 \mathrm{~min}$, islets were collected manually. Insulin secretion was measured from islets incubated with $95 \%$ air $/ 5 \% \mathrm{CO}_{2}$ at $37^{\circ} \mathrm{C}$ using a temperature-controlled multi-chamber perifusion system [23]. Groups of 50 islets were placed in four parallel perifusion chambers. The perifusion system used two computer-controlled peristaltic pumps to allow the perfusate glucose concentration to increase progressively from 2.8 to $16.7 \mathrm{mmol} / 1$ over $60 \mathrm{~min}$. Insulin concentrations were measured in the effluent perfusate every $\min$.

In situ pancreatic perfusions. Mice were anaesthetized and prepared for pancreas perfusion as described elsewhere [24]. The pancreas was perfused at $37^{\circ} \mathrm{C}$ and maintained at $1.5 \mathrm{ml} / \mathrm{min}$. The pancreatic effluent of the first $30 \mathrm{~min}$ of perfusion with basal glucose $(2.8 \mathrm{mmol} / \mathrm{l})$ was not collected. Following this equilibration period, the effluent was collected in 1-min intervals from a catheter placed in the portal vein. The insulin content of each interval was determined by ELISA.

Mitochondrial activity of pancreatic islet cells. Pancreatic islets were isolated from male mice by the collagenase technique [22], the islets were hand picked and 30 to 50 islets were used per experiment. Oxygen utilization was measured polarographically with a Clark oxygen electrode in a micro-water-jacketed cell at $37^{\circ} \mathrm{C}$ (Hansatech Instruments, Norfolk, UK) [25]. We measured the cell respiration in digitonin-permeabilized islets using pyruvate $(10 \mathrm{mmol} / \mathrm{l})$ as metabolic substrate to measure the activity of the complex I, succinate $(10 \mathrm{mmol} / \mathrm{l})$ for complex II, and glycerol-3-phosphate $(20 \mathrm{mmol} / \mathrm{l})$ for complex III.

Morphometric analysis of the islets. Serial sections were cut from each pancreas block and immunostained for insulin using the indirect peroxidase technique, anti-serum to insulin (Dako, Glöstrup, Denmark) was used at a dilution of 1/10. The islet surface was calculated after $o$-toluidine staining. Morphometry was carried out using a manual optical picture image analyzer (Model MOP-01, Olympus, Tokyo, Japan) on a projected image of the histological sections of the pancreas [26].

Apoptosis detection. Apoptosis was detected on paraffin-embedded pancreatic tissue by TUNEL method using the ApoAlert DNA Fragmentation Assay Kit (Clontech Laboratories, Palo Alto, Calif., USA). After apoptosis procedure, samples were immunostained for insulin with guinea pig anti-insulin antibody (1/200 dilution, ICN Pharmaceuticals, Costa Mesa, Calif., USA).

Statistical analysis. All statistical procedures were done using SSPS statistical software. Data analysis was carried out using the Kruskal-Wallis 1-way ANOVA. When interactions were significant, differences between individual group means were 
Table 1. Physical and blood parameters of $m G P D H^{--}$knockout animals fed with either standard or high carbohydrate diet for 1 month

\begin{tabular}{|c|c|c|c|c|c|c|}
\hline & \multicolumn{3}{|c|}{ Standard diet } & \multicolumn{3}{|c|}{ High carbohydrate diet } \\
\hline & WT (+/+) & $\operatorname{HET}(+/-)$ & HOMO (-/-) & WT (+/+) & HET (+/-) & $\operatorname{HOMO}(-/-)$ \\
\hline Food intake & $156 \pm 2$ & $157 \pm 3$ & $141 \pm 5^{\mathrm{a}}$ & $119 \pm 5^{d}$ & $123 \pm 2^{d}$ & $119 \pm 6^{\mathrm{d}}$ \\
\hline Energy (Kcal/day/Kg mice) & $494 \pm 7$ & $499 \pm 10$ & $447 \pm 14^{\mathrm{a}}$ & $471 \pm 18$ & $491 \pm 9$ & $474 \pm 24$ \\
\hline Glycaemia $(\mathrm{mmol} / \mathrm{l})$ & $7.4 \pm 0.3$ & $7.5 \pm 0.4$ & $7.8 \pm 0.5$ & $8.6 \pm 0.4^{c}$ & $8.5 \pm 0.4^{c}$ & $6.9 \pm 0.2^{\mathrm{a}}$ \\
\hline $\mathrm{TG}(\mathrm{mmol} / \mathrm{l})$ & $0.78 \pm 0.09$ & $0.78 \pm 0.14$ & $1.17 \pm 0.13^{\mathrm{a}}$ & $0.51 \pm 0.06^{c}$ & $0.37 \pm 0.04^{c}$ & $0.64 \pm 0.13^{c}$ \\
\hline Cholesterol (mmol/l) & $1.54 \pm 0.13$ & $1.85 \pm 0.13$ & $2.06 \pm 0.11^{b}$ & $2.57 \pm 0.13^{\mathrm{c}}$ & $2.23 \pm 0.35$ & $2.35 \pm 0.11$ \\
\hline $\mathrm{T} 3(\mathrm{pmol} / \mathrm{l})$ & $7.78 \pm 0.38$ & $8.98 \pm 0.77$ & $7.34 \pm 0.28$ & $10.13 \pm 0.64^{d}$ & $10.10 \pm 0.95^{\mathrm{c}}$ & $10.63 \pm 0.40^{\mathrm{d}}$ \\
\hline
\end{tabular}

Results are expressed as the means \pm SEM for at least 16 animals of each genotype. ${ }^{a} p<0.05$ as compared to wild-type animals from the same diet, ${ }^{b} p<0.01$ as compared to wild-type an-

analyzed by Student's $t$ test. A $p$ value of less than 0.05 was considered statistically significant.

\section{Results}

Physical and plasma measurements. The mice were fed with a high carbohydrate diet, and, after 1 month of treatment, wild-type and heterozygous mice showed mild hyperglycaemia. However, in $m G P D H^{-1-}$ mice, the high carbohydrate diet did not increase the glycaemia; they remained normoglycaemic, as did the mice fed with the standard diet (Table 1). Moreover, incidence of diabetic values $(>11 \mathrm{mmol} / \mathrm{l})$ was higher in $\mathrm{mGPDH}^{+/+}(20 \%)$ and $m G P D H^{+/-}(11 \%)$ than in $m G P D H^{-/}(0 \%)$ (Fig. 1A).

Body weight gain was similar in the mice fed with the high carbohydrate diet and those fed the standard diet, with the notable exception of the $m G P D H^{-/}$ mice, which grew by around $60 \%$ when fed with the high carbohydrate diet. This diet also induced hyperinsulinaemia in the wild-type and heterozygous mice, whereas in $m G P D H^{-/}$the insulin values were similar to those of the mice fed with the standard diet. It is worth noting that despite the differences in food intake, all the mice on the high carbohydrate diet and the standard one had similar caloric intake (Table 1).

The high carbohydrate diet had no significant effect on NEFA blood concentrations in wild type and heterozygous mice. However in $\mathrm{mGPDH}^{-/}$mice the decrease was pronounced. The $m G P D H^{-/-}$mice had higher cholesterol and triglyceride concentrations when fed with the standard diet (Table 1). The high carbohydrate diet induced a decrease in triglycerides and an increase in cholesterol in all the mice without differences between the three genotypes. T3 values were also increased in all the mice fed with the high carbohydrate diet; there were no differences with regard to genotype (Table 1). imals from the same diet, ${ }^{c} p<0.05$ as compared to the same experimental group with the standard diet, ${ }^{\mathrm{d}} p<0.01$ as compared to the same experimental group with the standard diet
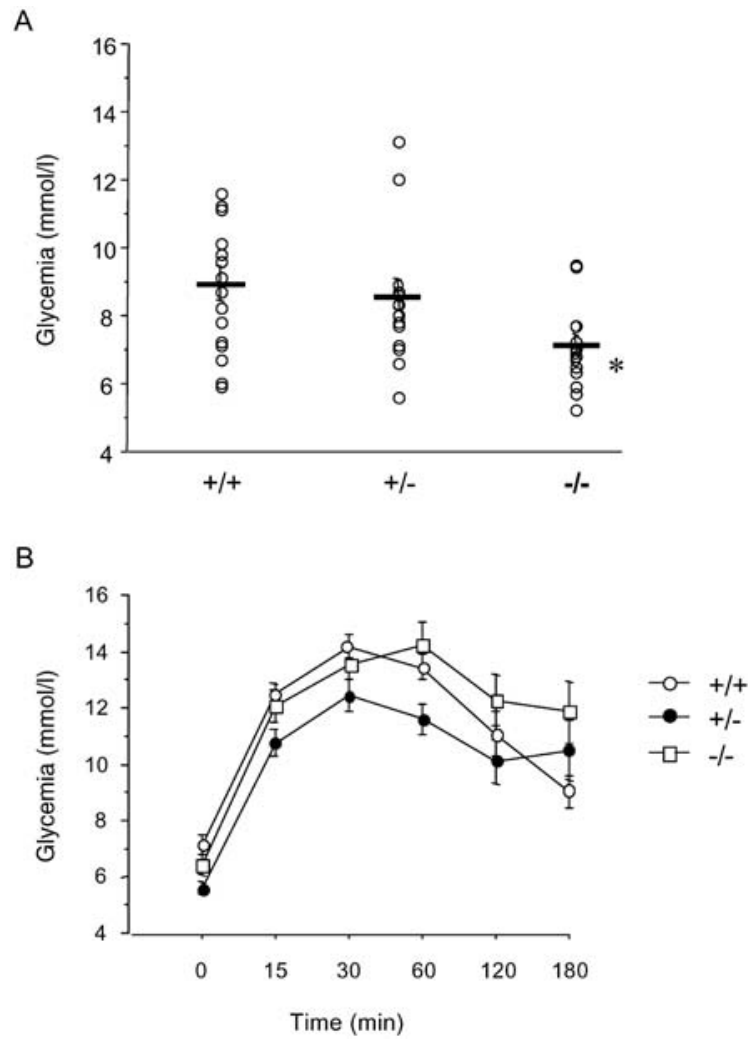

Fig. 1A, B. Glycaemia and intraperitoneal glucose tolerance test of $m G P D H^{+/+}, m G P D H^{+/-}$and $m G P D H^{-/-}$mice fed with a high carbohydrate diet for 1 month. (A) Blood glucose concentrations of wild-type $(n=15), \mathrm{mGPDH}^{+/-}(n=18)$ and $\mathrm{mGPDH}^{-/-}$ $(n=18)$ mice. Each value is represented as an empty circle. The mean for each group is drawn as a bar with the SEM. *, $p<0.05$ for $\mathrm{mGPDH}^{-1-}$ mice compared to the other two groups. (B) Glucose tolerance test was done in the $m G P D H^{-1}$ mice as described. Results are expressed as mean blood glucose concentration \pm SEM from at least 16 mice of each genotype 


\section{Standard diet High carbohydrate diet}

A

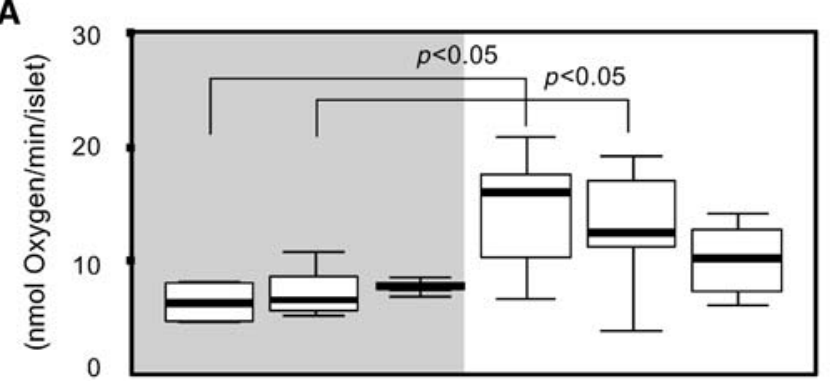

B

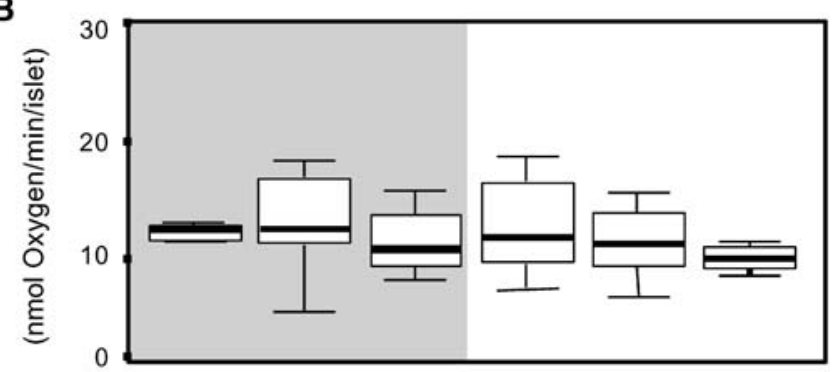

C

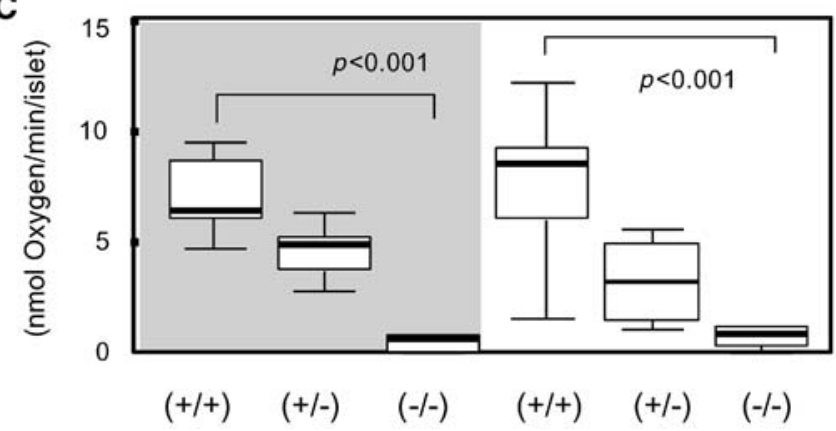

Fig. 2A-C. Respiratory activity of $m G P D H^{-/-}$islets. (A) Complex (I) Substrate pyruvate; (B) Complex (II) Substrate: Succinate; (C) Complex (III) Substrate: Glycerol 3-phosphate. The respiratory activity of the three complexes was measured from islets of $m G P D H^{+/+}, m G P D H^{+/-}$and $m G P D H^{-/}$mice fed with a high carbohydrate diet for 1 month. Results are expressed as BoxPlot, with the median as the horizontal line inside the box, from six independent experiments for each genotype. The $p$ values between different significant groups are plotted in the graph

Despite the differences observed in glucose and insulin values of $m G P D H^{-/}$mice fed with the high carbohydrate diet, the glucose tolerance test revealed no significant differences between the three groups (Fig. 1B). The test also did not detect differences in insulin values (data not shown).

Mitochondrial respiratory chain activity. Since the glycerol-phosphate shuttle is linked to the mitochondrial respiratory chain, we analyzed the oxygen consumption of the mitochondrial respiratory chain induced by various substrates. Oxidation of pyruvate (electrons entering complex I) was higher in the wildtype mice fed with the high carbohydrate diet; howev-

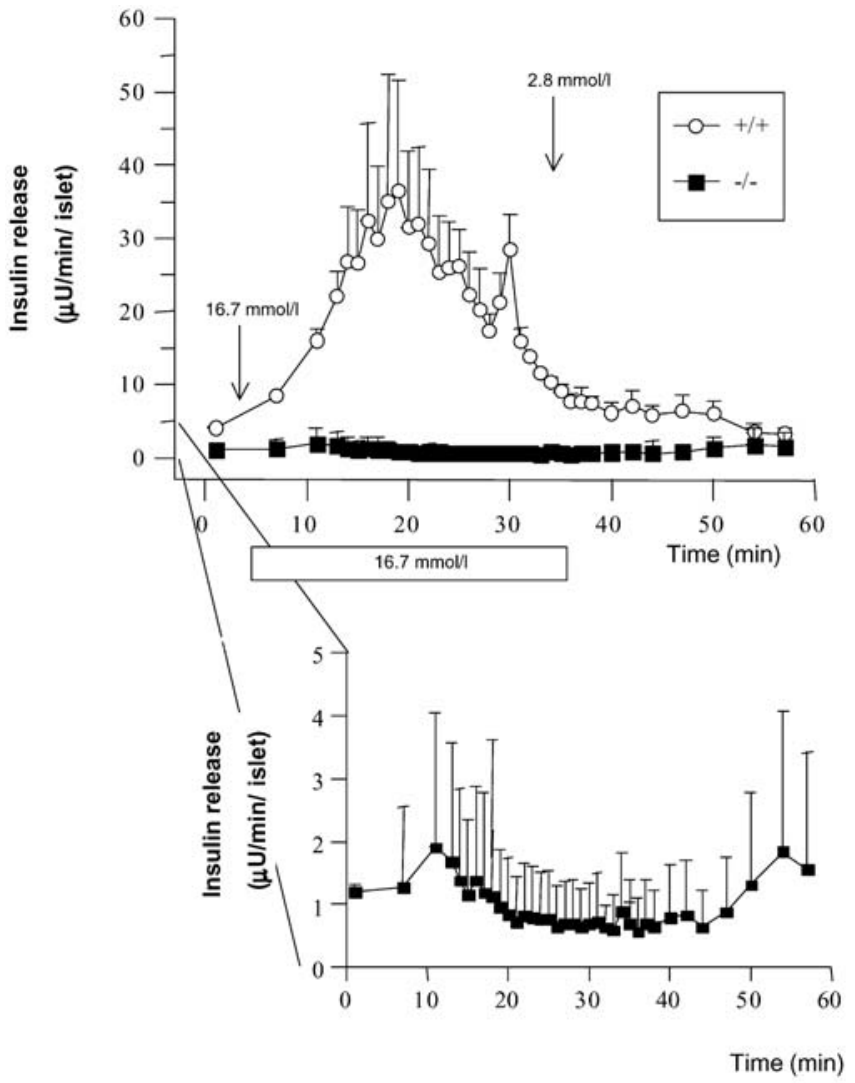

Fig. 3. Study of glucose-induced insulin secretion in $m G P D H^{-/-}$ islets. Insulin released from islet in perifusion obtained from $m G P D H^{+/+}$and $m G P D H^{-/-}$mice fed with high carbohydrate diet. Glucose concentration was raised from 2.8 to $16.7 \mathrm{mmol} / \mathrm{l}$ at $5 \mathrm{~min}$, and then decreased to $2.8 \mathrm{mmol} / \mathrm{l}$ at $35 \mathrm{~min}$. Data are from six independent experiments with islets from each genotype

er, in $m G P D H^{-/-}$mice the high carbohydrate diet did not induce an increase in oxygen consumption (Fig. 2). There were no differences in the oxidation of succinate (electrons entering complex II) between the two diets, or the different genotypes. Finally, oxygen consumption of mitochondria with glycerol-3-phosphate (electrons entering complex III) was decreased in $m \mathrm{GPDH}^{-/-}$mice regardless of diet (Fig. 2).

Islet function. In order to improve our understanding of islet function, we carried out islet perifusion experiments. An increase in insulin secretion was observed in the islets from wild-type mice fed with the high carbohydrate diet when glucose was augmented from $2.8 \mathrm{mmol} / \mathrm{l}$ to $16.7 \mathrm{mmol} / \mathrm{l}$ (Fig. 3). When glucose was decreased back to $2.8 \mathrm{mmol} / \mathrm{l}$, the insulin secretion fell to basal values. However, in $m G P D H^{-/-}$mice, no increase in insulin secretion was observed. In these mice, insulin release remained at baseline values throughout the experiment at different glucose concentrations (Fig. 3). Similar results were obtained in the in situ perfused pancreas preparation. The $m G P D H^{-/}$mice had a lower secretion rate compared 


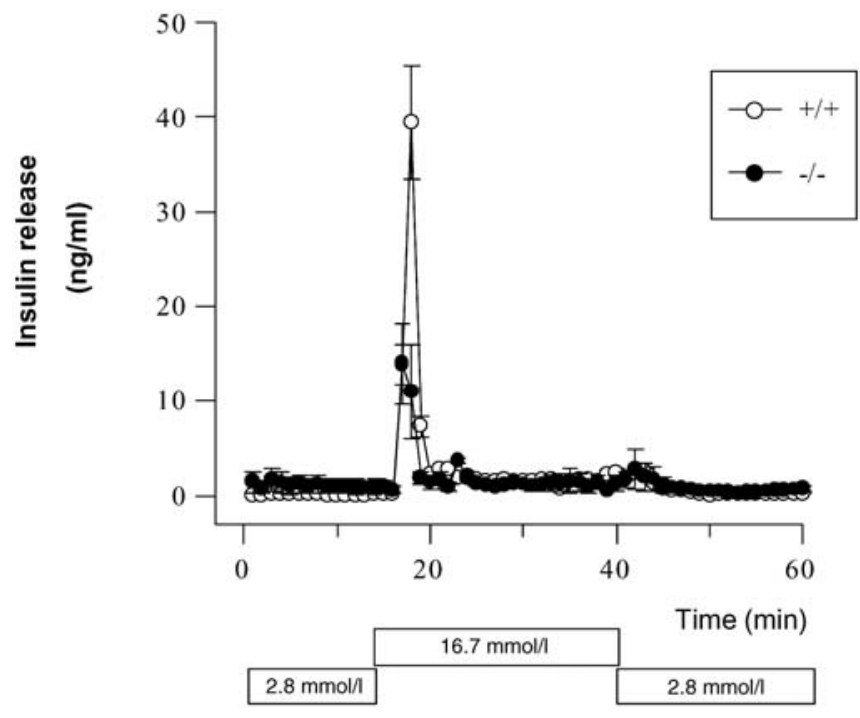

Fig. 4. Insulin secretion from in situ pancreatic perfusion. Insulin released from perfused pancreas of wild type and $m G P D H^{-1-}$ mice fed with high carbohydrate diet. Glucose concentration was raised from 2.8 to $16.7 \mathrm{mmol} / \mathrm{l}$ at $16 \mathrm{~min}$, and then decreased to $2.8 \mathrm{mmol} / \mathrm{l}$ at $40 \mathrm{~min}$. Results are from two independent experiments

to wild-type mice when glucose was shifted from 2.8 to $16.7 \mathrm{mmol} / \mathrm{l}$, despite an increase in the secretory response (Fig. 4).

Long-term high carbohydrate diet. After analyzing the results obtained from the 1-month period on the high carbohydrate diet, we investigated the effects of the diet over a longer period ( 6 months). The blood glucose concentrations increased in the $m G P D H^{+/+}$and $m G P D H^{+/}$ mice fed with the high carbohydrate diet. After 3 months of diet, the values tended to decrease, reaching values similar to the ones at the beginning of the experiment. However, $m G P D H^{--}$mice had lower glucose concentrations than the $m G P D H^{+/+}$and $m G P D H^{+/}$ mice throughout the 6-month period (Fig. 5A).

The body weight of the wild-type and heterozygous mice increased during the 6 months on the high carbohydrate diet; in contrast, the body weight of $m G P D H^{-/}$ mice showed a smaller increase during the 6 months of diet (Fig 5B). The mice fed with standard diet showed a similar tendency, the $m G P D H^{-/-}$mice being the ones with lower body weights after 6 months with standard diet $\left(38.7 \pm 3.2\right.$ for $m G P D H^{+/+}, \quad 39.3 \pm 2.7$ for $m G P D H^{+-}$, and $29.9 \pm 0.8$ for $m G P D H^{--}$). The mice fed with the standard diet had a higher body weight increase than the ones fed with a high carbohydrate diet.

The results of the glucose tolerance test were similar to those obtained after 1 month. No differences were observed in the blood glucose and insulin concentrations between the different genotypes during the test (data not shown). In order to assess the insulin resistance of the mice, we carried out an insulin tolerance test. In the wild-type mice, the administration of
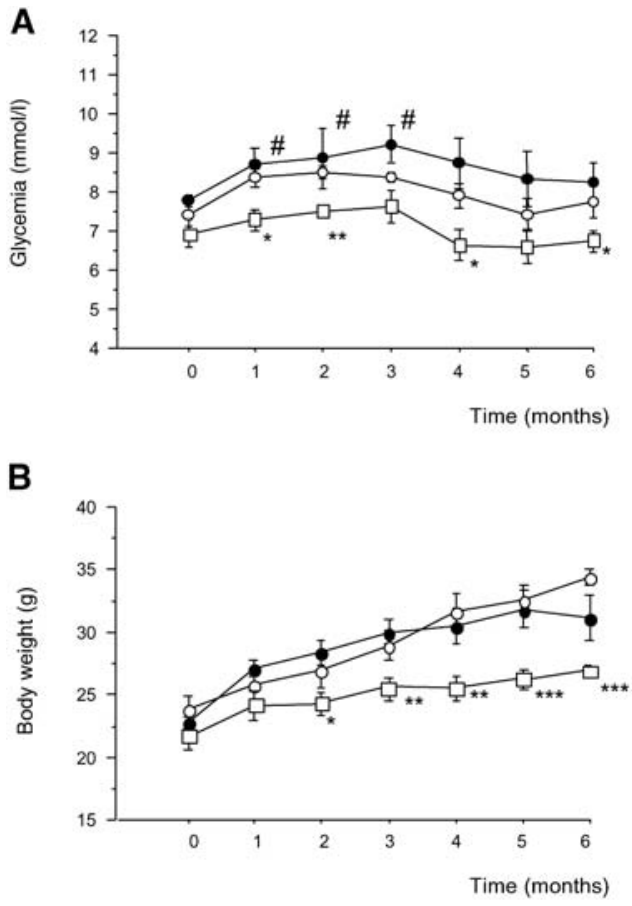

Fig. 5A, B. Glycaemia and body weight evolution of $m G P D H^{-/-}$mice fed with a carbohydrate diet for 6 months. (A) Evolution of blood glucose concentrations during the diet. (B) Body weight evolution of the mice. Results are expressed as means \pm SEM from at least six mice of each genotype. $* p<0.05$; ** $p<0.01$; *** $p<0.001$ for $\mathrm{mGPDH}^{-1-}$ mice compared to the other two groups. ${ }^{\#} p<0.05$ for wild-type, $\mathrm{mGPDH}^{+/-}$mice compared to the value at time zero

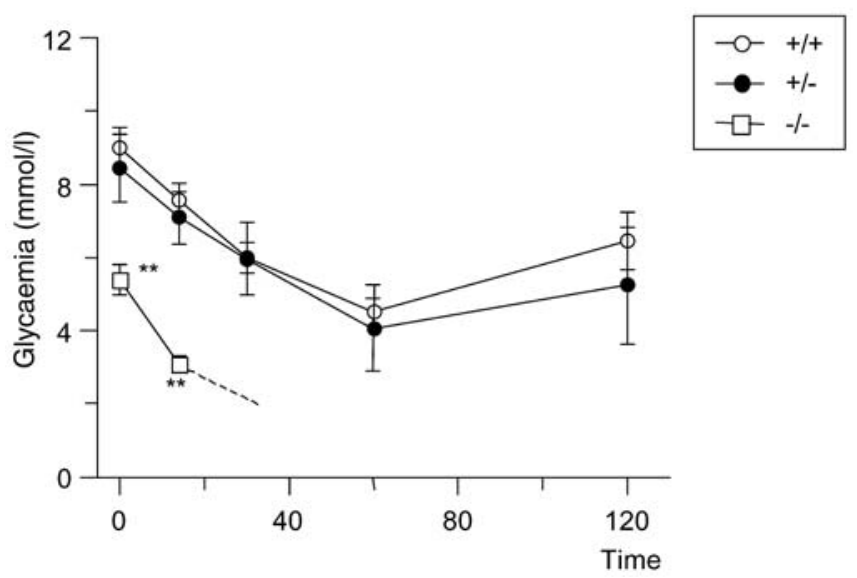

Fig. 6. Insulin tolerance test of $m G P D H^{-/-}$mice fed with a carbohydrate diet for 6 months. Results are expressed as mean blood glucose concentration \pm SEM from at least five mice of each genotype. ${ }^{*} p<0.01$ for $m G P D H^{--}$mice compared to the other two groups. The $m G P D H^{-/-}$mice had very low glycaemic values and they did not survive the test

insulin decreased the glucose concentrations, as expected (Fig. 6). Surprisingly, $m G P D H^{-/}$mice had a lower fasting glycaemia, and the administration of the insulin produced relatively severe hypoglycaemia leading to death in some cases. The heterozygous mice presented an intermediate pattern. 

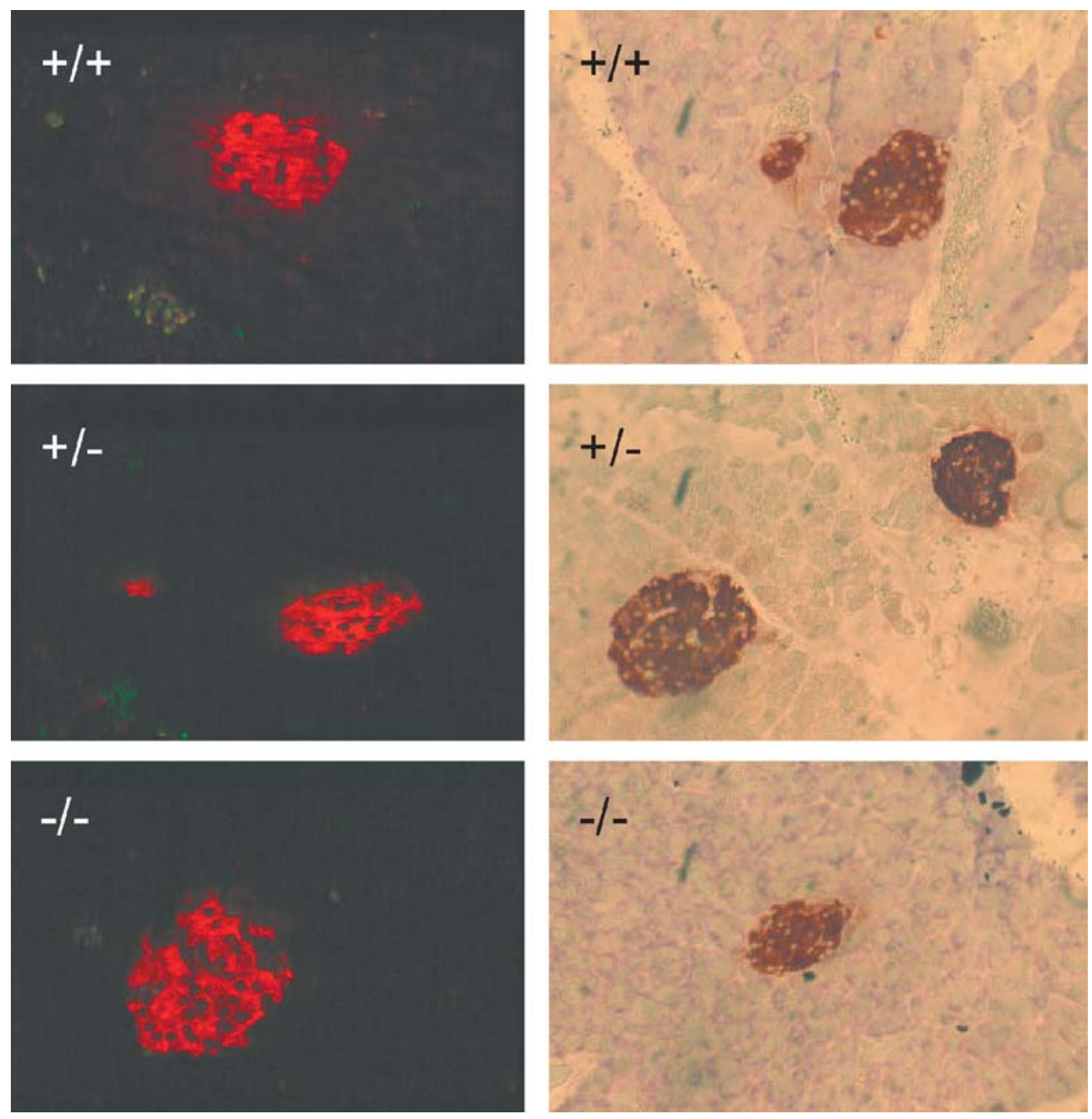

Fig. 7. Morphology and apoptosis in islets from $m G P D H^{-1}$ mice fed with a carbohydrate diet for 6 months. Representative images of the apoptosis (left side, DNA fragmentation in green and insulin in red) and morphology (right side, stained with insulin) of the three genotypes after the diet treatment $(\times 40)$

Table 2. Morphometric analysis of pancreatic islets of the mice fed with high carbohydrate diet

\begin{tabular}{llll} 
Islet morphology & & & \\
\hline & WT $(+/+)$ & HET $(+/-)$ & HOMO $(-/-)$ \\
\hline Beta cell/pancreas $(\%)$ & $7.7 \pm 0.8$ & $6.9 \pm 0.9$ & $4.9 \pm 0.7^{a}$ \\
Islet area $\left(\mu \mathrm{m}^{2}\right)$ & $7471 \pm 715$ & $6252 \pm 1352$ & $5964 \pm 999$
\end{tabular}

Results are expressed as means \pm SEM for four mice of each genotype

${ }^{\text {a }} p<0.05$ compared to wild-type mice

The morphometric analysis of the pancreas of the mice showed that the high carbohydrate diet induced a hyperplasia of the islets in all three genotypes, while the pancreas weight did not change (Table 2, Fig. 7). However, the percentage of beta cells in the pancreas

was lower in the $m G P D H^{-/-}$mice. Moreover, no significant differences between the three genotypes were observed in the apoptosis rate (Fig. 7), nor in glycogen and lipid content in the liver. There were no variations in the epididymal adipose tissue weight.

\section{Discussion}

Pancreatic islet beta cells are poised to generate metabolic messengers in the mitochondria that link glucose metabolism to insulin exocytosis. The mGPDH is the rate-limiting enzyme of the glycerol phosphate shuttle which, together with the malate-aspartate shuttle, permits the reoxidation of cytosolic NADH. mGPDH is particularly abundant in pancreatic islets [2] and, in contrast to lactate dehydrogenase, much more so in beta cells than in non-beta cells [27]. Mice with a targeted disruption of $m G P D H$ have recently been generated and found to be apparently normal. In this paper, using $m G P D H$ knockout mice, we first hypothesized that an environmental factor, such as a high carbohydrate diet, would compromise the mitochondrial metabolism and, consequently, insulin secretion, thus rendering these mice diabetic. Surprisingly, these mice 
did not become diabetic, but showed lower blood glucose and insulin concentrations compared to the wildtype mice.

The results obtained help to further characterize $m G P D H$ mice. Although several previous reports have described $m G P D H^{-/}$mice as normal $[14,15,16,17]$, our results showed that the blood concentrations of triglycerides and cholesterol in $\mathrm{mGPDH}^{-/}$mice are increased. These results could be due to the absence of mGPDH activity in the liver, but not in the pancreas. Tough liver has lower concentrations of mGPDH compared to beta cell; in $m G P D H^{-/-}$mice, lower concentrations of liver ATP and glycerol phosphate has been found recently [15]. Therefore, the lack of $m G P D H^{-}$could modify the NADH shuttle in the liver altering lipid metabolism. In $\mathrm{mGPDH}^{-/}$mice, the NEFA concentrations remain unchanged [15], which is consistent with our findings.

The results clearly show that the diet induced hyperglycaemia and hyperinsulinaemia in wild-type and heterozygous mice. The high carbohydrate diet decreased blood triglycerides in these mice, while NEFA remained unchanged; this could be due, at least partially, to the higher protein content of the high carbohydrate diet, since the fat content is the same. Moreover, some studies have also shown that high carbohydrate diets could decrease triglyceride concentrations [28]. The increased respiratory activity of mitochondrial respiratory chain with complex I substrate observed in the $\mathrm{mGPDH}^{+/+}$and $\mathrm{mGPDH} \mathrm{H}^{+/-}$mice probably results from an increased glycolytic rate, since electron transport activity can adapt to the energy status of the cell [29]. This increased flow through the mitochondrial respiratory chain could increase the ATP/ADP ratio leading to higher insulin secretion, as found in the mice. Moreover, the mice have beta-cell hyperplasia in the pancreas. Therefore, all these changes seem to represent an adaptation mechanism to the high carbohydrate intake.

In the $\mathrm{mGPDH}^{-/-}$mice, the high carbohydrate diet did not induce the same changes observed in the $m \mathrm{GPDH}^{+/+}$and $m G P D H^{+/-}$mice. The $m G P D H^{-/-}$ mice had lower glycaemia and insulinaemia than the wild-type and heterozygous mice, and a lower betacell percentage in the pancreas, which is not due to an increased apoptotic rate. The $m G P D H^{-/}$mice fed with the high carbohydrate diet had a higher sensitivity to insulin, due, at least partially, to the lower NEFA concentrations. The glucose-induced insulin secretion was also lower in perifused islets and perfused pancreas. It is difficult to reconcile the normal insulin response in the whole animal during the glucose tolerance test and the impaired glucose induced insulin secretion. When we study the response of an isolated organ or some cells, regulatory factors are lost as the model is simplified. For example, the isolated islets show a biphasic secretion while the perfused pancreas only shows a monophasic secretion; while these data are well known, the explanation remains unknown [30]. For some reason, in the high carbohydrate-fed $m \mathrm{GPDH}^{-/}$mice, the insulin response to glucose seems normal in whole mice, perhaps due to a diminished insulin clearance from the liver; notwithstanding, the isolated islets and the pancreas show an impaired response.

The lower body weight showed by the $m G P D H^{-/-}$ mice was already reported in the papers describing the $m G P D H$ knockouts $[14,15]$, and our data agree with the percentage reported in those papers. Moreover, the high carbohydrate diet induces an increase in the thermogenesis, and the mice show a lower weight gain compared to standard diet [31], similar to what we observed in our experiments. Although it has been reported that, with standard diet, the thermogenesis was not altered in the $m G P D H^{-/}$mice [15], we cannot exclude that the lack of $m G P D H$ alters the thermogenesis induced by the high carbohydrate diet, and enhances the body weight differences. The contribution of the brown adipose tissue or other peripheral tissues was not analyzed. It has been reported that $m G P D H^{-/}$ mice fed with a high fat diet show smaller epididymal fat pads [15]; however, in our experiments, no difference was observed. These variations are probably due to the different composition of the diets; therefore only when the accumulation of lipids is greatly stimulated, i.e. high fat diets, the lack of $m G P D H$ results phenotipically in smaller fat accumulations.

In conclusion, the high carbohydrate diet-induced hyperglycaemia and hyperinsulinaemia in the wildtype and $m G P D H^{+/-}$mice. However, the $m G P D H^{-/-}$ fed with the high carbohydrate diet had a lower value of glycaemia and insulinaemia; and a decreased respiratory activity. Similar results were obtained when the $m G P D H^{-/}$mice were fed with a high fat diet (10\%), showing lower glucose concentrations compared to wild-type mice [15]. Moreover, these mice had increased insulin sensitivity. Therefore, the lack of $m G P D H$ acts as a rate-limiting step for glucose metabolism in the high carbohydrate fed $m G P D H^{-/}$ mice. Moreover, the high carbohydrate diet does not worsen the phenotype of the animal with a partial impairment of NADH shuttle in the mitochondria.

Acknowledgements. We would like to thank C.B. Wollheim and T. Kadowaki for their help and advice in designing the experiments and preparing the manuscript. This work was supported by grants SAF 97/0023 from Ministerio de Ciencia y Tecnología (Spain), Red de Centros C03/08, RGDM G03/212 and FIS 96/0164 from Ministerio de Sanidad y Consumo (Spain), and SGR 2001/00378 from Generalitat de Catalunya (Spain). A.B. was a postdoctoral fellow from Ministerio de Educacion (Spain). P.M. is a fellow from the Dr. Max Cloëtta Foundation (Zurich). We thank all the members of the Laboratory of Experimental Diabetes for the invaluable discussions and support, and M. Maudsley for assistance in preparing the English manuscript. 


\section{References}

1. Maechler P, Wollheim CB (2001) Mitochondrial functioning in normal and diabetic beta cells. Nature 414:807812

2. MacDonald MJ (1981) High content of mitochondrial glycerol-3-phosphate dehydrogenase in pancreatic islets and its inhibition by diazoxide. J Biol Chem 256:8287-8290

3. MacDonald MJ (1982) Evidence for the malate aspartate shuttle in pancreatic islets. Arch Biochem Biophys 213: 643-649

4. MacDonald MJ, Brown LJ (1996) Calcium activation of mitochondrial glycerol phosphate dehydrogenase restudied. Arch Biochem Biophys 326:79-84

5. Ishihara H, Nakazaki M, Kanegae Y et al. (1996) Effect of mitochondrial and/or cytosolic glycerol 3-phosphate dehydrogenase overexpression on glucose-stimulated insulin secretion from MIN6 and HIT cells. Diabetes 45:1238-1244

6. Giroix MH, Rasschaert J, Bailbe D et al. (1991) Impairment of the glycerol phosphate shuttle in islets from rats with diabetes induced by neonatal streptozotocin. Diabetes 40:227-232

7. Giroix MH, Baetens D, Rasschaert J et al. (1992) Enzymic and metabolic anomalies in islets of diabetic rats: relationship to beta cell mass. Endocrinology 130:2634-2640

8. Anak O, Giroix MH, Sener A, Malaisse WJ (1993) FADglycerophosphate dehydrogenase activity in splenocytes of rats with acquired or inherited diabetes mellitus. Med Sci Res 21:565-566

9. Giroix MH, Sener A, Portha B, Malaisse WJ (1993) Preferential alteration of oxidative relative to total glycolysis in pancreatic islets of two model of inherited and acquired type 2 (non insulin-dependent) diabetes mellitus. Diabetologia 36: 305-309

10. MacDonald MJ, Tang J, Polonsky KS (1996) Low mGDH and pyruvate carboxylase in pancreatic islets of Zucker Diabetic Fatty rats. Diabetes 45:1626-1630

11. Fernàndez-Alvarez $\mathrm{J}$, Conget $\mathrm{I}$, Rasschaert $\mathrm{J}$, Sener A, Gomis R, Malaisse WJ (1994) Enzymatic, metabolic and secretory patterns in human islets of type 2 (non-insulindependent) diabetic patients. Diabetologia 37:177-181

12. Malaisse WJ (1994) Physiology and pathology of islet metabolism. Diabetes Metab 20: 95-98

13. Brown LJ, Stoffel M, Moran SM et al. (1996) Structural organization and mapping of the human mitochondrial glycerol phosphate dehydrogenase-encoding gene and pseudogene. Gene 172:309-312

14. Eto K, Tsubamoto Y, Terauchi Y et al. (1999) Role of NADH shuttle system in glucose-induced activation of mitochondrial metabolism and insulin secretion. Science 283:981-985

15. Brown LJ, Koza RA, Everett C et al. (2002) Normal thyroid thermogenesis but reduced viability and adiposity in mice lacking the mitochondrial glycerol phosphate dehydrogenase. J Biol Chem 277:32892-32898
16. Eto K, Suga S, Wakui M et al. (1999) NADH shuttle system regulates $\mathrm{K}_{\text {ATP }}$ channel-dependent pathway and steps distal to cytosolic $\mathrm{Ca}^{2+}$ concentration elevation in glucose-induced insulin secretion. J Biol Chem 274: 25386-25392

17. Ravier MA, Eto K, Jonkers FC, Nenquin M, Kadowaki T, Henquin JC (2000) The oscillatory behavior of pancreatic islets from mice with mitochondrial glycerol-3-phosphate dehydrogenase knockout. J Biol Chem 275:1587-1593

18. Taylor SI (1999) Deconstructing type 2 diabetes. Cell 97:9-12

19. Saltiel AR (2001) New perspectives into the molecular pathogenesis and treatment of type 2 diabetes. Cell 104: 517-529

20. Miyawaki K, Yamada Y, Yano H et al. (1999) Glucose intolerance caused by a defect in the entero-insular axis: a study in gastric inhibitory polypeptide receptor knockout mice. Proc Natl Acad Sci USA 96:14843-14847

21. Seino S, Iwanaga T, Nagadhima K, Miki T (2000) Diverse roles of $\mathrm{K}_{\mathrm{ATP}}$ channels learned from Kir6.2 genetically engineered mice. Diabetes 49:311-318

22. Lacy PE, Kostianovsky M (1967) Method for the isolation of intact islets of Langerhans from the rat pancreas. Diabetes 16:35-39

23. Malaisse-Lagae F, Malaisse WJ (1984) Insulin release by pancreatic islets. In: Larner J, Pohl SL (eds) Methods in diabetes research. Wiley, New York, pp 147-152

24. Maechler P, Gjinovci A, Wollheim CB (2000) Implication of glutamate in the kinetics of insulin secretion in rat and mouse perfused pancreas. Diabetes 51(Suppl 1):S99-S102

25. Conget I, Barrientos A, Manzanares JM et al. (1997) Respiratory chain activity and mitochondrial DNA content of nonpurified and purified pancreatic islets cells. Metabolism 46:984-987

26. Clark A, Wells CA, Buley ID et al. (1988) Islet amyloid, increased A-cells, reduced B-cells and exocrine fibrosis: quantitative changes in the pancreas in type 2 diabetes. Diabetes Res 9:151-159

27. Sekine N, Cirulli V, Regazzi R et al. (1994) Low lactate dehydrogenase and high mitochondrial glycerol phosphate dehydrogenase in pancreatic beta-cells. Potential role in nutrient sensing. J Biol Chem 269:4895-4902

28. Riccardi G, Rivellese AA (2000) Dietary treatment of the metabolic syndrome-the optimal diet. Br J Nutr 83: S143-S148

29. Civelek VN, Deeney JT, Shalosky NJ et al. (1996) Regulation of pancreatic beta-cell mitochondrial metabolism: influence of $\mathrm{Ca} 2+$, substrate and ADP. Biochem J 318: 615-621

30. Nesher R, Cerasi E (2002) Modeling phasic insulin release. Immediate and time-dependent effects of glucose. Diabetes 51:S53-S59

31. Rothwell NJ, Stock MJ, Warwick BP (1983) The effects of high fat and high carbohydrate cafeteria diets on diet-induced thermogenesis in rat. Int J Obes 7:263-270 